\title{
Relationships of Tarentola (Reptilia: Gekkonidae) from the Cape Verde Islands estimated from DNA sequence data
}

\author{
José Jesus ${ }^{1}$, António Brehm¹ , D. James Harris ${ }^{2}$ \\ ${ }^{1}$ Centro de Ciências Biológicas e Geológicas, University of Madeira, Penteada, 9000 Funchal, Portugal \\ ${ }^{2}$ Centro de Estudos de Ciência Animal (CECA), ICETA-U.P., Campus Agrário de Vairão, 4485-661 Vila do \\ Conde, Portugal \\ e-mail: james@mail.icav.up.pt
}

\begin{abstract}
Thirteen specimens of Tarentola from the Cape Verde islands were sequenced for 695 base pairs of $12 S$ rRNA and cytochrome $b$ mitochondrial genes, and analysed with published sequences. Our results support many of the relationships previously proposed. We report the presence of Tarentola gigas Bocage, 1875 on São Nicolau and Tarentola caboverdiana nicolauensis Schleich, 1984 on São Vicente. This increases the number of genetically distinct forms on these islands; hence community structure appears to be more complex than previously understood. We also sequenced seven individuals for 375 base pairs of the nuclear gene, C-mos. Two sites were variable, much less than expected given the high levels of differentiation based on mitochondrial DNA sequences.
\end{abstract}

\section{Introduction}

The Cape Verde archipelago, approximately $400 \mathrm{~km}$ from the West African coast, comprises ten islands and a number of islets of volcanic origin (Mitchell-Thomé, 1983). The islands form an arch (fig. 1), the oldest islands being Maio, Sal and Boavista, and the youngest Santo Antão and Brava, the tips of the "arch". During sea level fluctuations in the Pleistocene some of the northwestern group of islands were most likely linked — São Vicente, Santa Luzia, Branco and Raso, and possibly also Boavista and Maio from the "central" group of islands. Otherwise the water channels are very deep (>1000 meters) and so it is highly improbable that these islands were ever linked by land connections. DNA sequence data have recently been used to estimate colonization patterns of reptiles within the Cape Verde islands (Brehm et al., 2001; Carranza et al., 2001; Jesus et al., 2001). In the case of Tarentola a species of the subgenus Makariogecko appears to have colonized the Cape Verde islands from Gomera or Tenerife in the Western Canaries (Joger, 1984; 


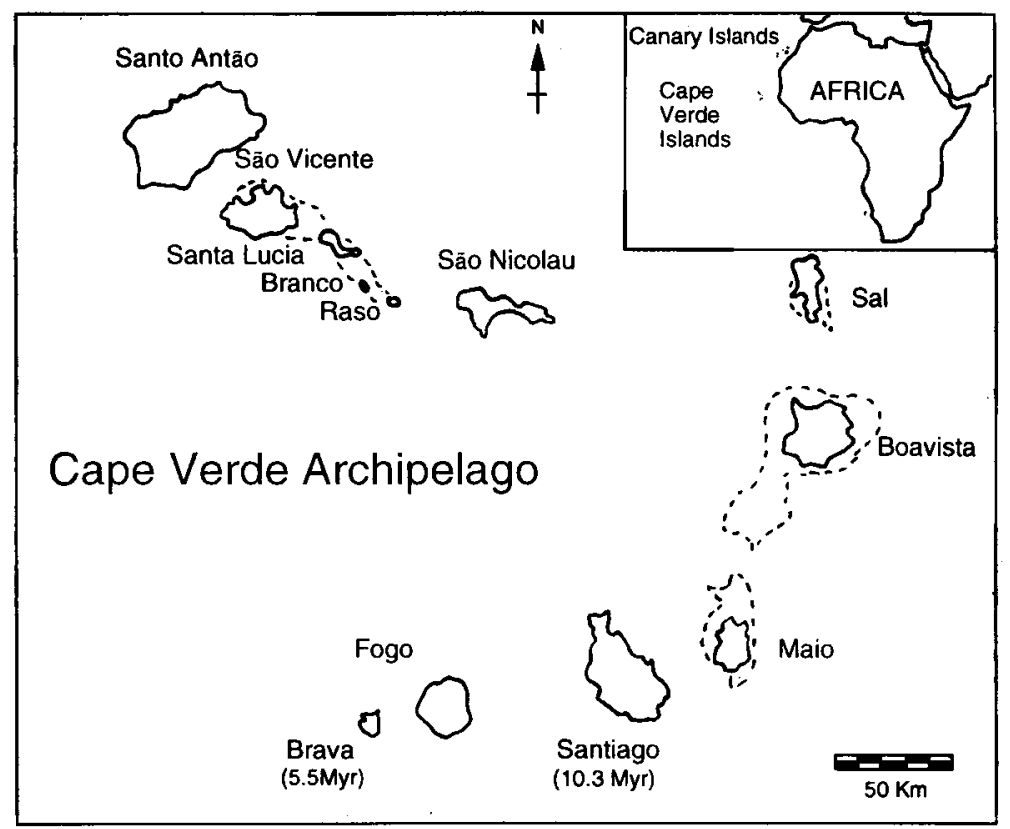

Figure 1. Map of the Cape Verde islands. Localities of specimens used in this study are given in table 1. Shallow water areas, which were probably above sea level in the geologically recent past, are marked with dashed lines.

Carranza et al., 2000). Nine genetically distinct units were defined from the Cape Verde islands on the basis of partial 12S rRNA and cytochrome $b$ mitochondrial DNA (mtDNA) sequences (Carranza et al., 2000). Here we combine data from the same genes for 13 additional individual Tarentola from the islands, and also sequences from the nuclear gene C-mos to compare with the mtDNA data. Inclusion of our new data does not alter many of the taxonomic conclusions of Carranza et al. (2000). Carranza et al. (2000), however, reported only a single species of Tarentola from each of the northern islands except São Nicolau and the islets of Branco and Raso. We found additional species on the larger islands in the northern group. Although Tarentola show higher levels of mtDNA variation within the Cape Verde islands compared to Mabuya skinks (Brehm et al., 2001; Carranza et al., 2001), they show lower levels of variation in the nuclear gene C-mos. Despite the extensive sampling of Carranza et al. (2000) the genetic variation of Tarentola within the Cape Verde islands is more complex than previously described.

\section{Methods}

Additional specimens examined are shown in table 1. Specimens collected in the field were identified following Joger (1993) and Schleich (1987), and released after tail tips were taken. Tissues are preserved in the collections of CCBG (University of Madeira). The sample from T. c. raziana Schleich, 1984 (from Raso island) comes from the collection of the Center of Zoology (IICT, Lisbon). Total genomic DNA was extracted from small pieces 
Table 1. Specimens included in the mtDNA analysis with locality and specimen voucher number.

\begin{tabular}{|c|c|c|}
\hline Specimen & Island (locality) & Voucher \\
\hline T. caboverdiana nicolauensis & São Nicolau (Ponta Cachorro) & T23848 \\
\hline T. caboverdiana nicolauensis & São Nicolau (Ribeira Brava) & T23849* \\
\hline T. caboverdiana nicolauensis & São Nicolau (Ribeira Brava) & $\mathrm{T} 23847$ \\
\hline T. caboverdiana nicolauensis & São Vicente (Mindelo) & $\mathrm{T} 23850^{*}$ \\
\hline T. caboverdiana nicolauensis & São Vicente (Mindelo) & $\mathrm{T} 23851$ \\
\hline T. caboverdiana caboverdiana & Santo Antão (Porto Novo) & T23839 \\
\hline T. caboverdiana caboverdiana & Santo Antão (Ponta do Sol) & $\mathrm{T} 23855$ \\
\hline T. caboverdiana substituta & São Vicente (Madeiral) & $\mathrm{T} 23891$ \\
\hline T. caboverdiana substituta & São Vicente (Madeiral) & $\mathrm{T} 23892$ \\
\hline T. caboverdiana raziana & Razo & ПСТ $317^{*}$ \\
\hline T. gigas & São Nicolau (Ponta Cachorro) & T23893* \\
\hline T. darwini & Santiago (Tarrafal) & $\mathrm{T} 23894$ \\
\hline T. darwini & Fogo (São Filipe) & T23895 \\
\hline
\end{tabular}

${ }^{*}$ Indicate the partial C-mos region was also sequenced. Three other specimens were also sequenced for C-mos (T. rudis and two T. darwini, all from Santiago, codes T23882, T23897 and T23898 respectively).

of tail using standard methods. Polymerase Chain Reaction primers used in both amplification and sequencing were cytochrome $b 1$ and $b 2$ and 12Sa and 12Sb from Kocher et al. (1989) and G73 and G74 for C-mos from Saint et al. (1998). Amplification conditions were the same as described by Harris et al. (1998) and Saint et al. (1998). Amplified fragments were sequenced from both strands on a 373 Applied Biosystem DNA Sequencing Apparatus.

Sequence analysis. Sequences from the Cytochrome $b$ and 12S rRNA belonging to the same individual were merged for the analysis. These and the C-mos sequences were aligned using Clustal W (Thompson et al., 1994) with the previously published data (Carranza et al., 2000; Harris et al., 2001). Three samples of Tarentola gomerensis Joger and Bischoff, 1983 were designated as outgroup taxon. Cytochrome $b, 12 \mathrm{~S}$ rRNA and C-mos sequences were respectively 303,392 and 375 base pairs long. GenBank accession numbers are AF468790 to AF468815. The cytochrome $b$ and C-mos sequences contained no indels.

The data were imported into PAUP* 4.0b5 (Swofford, 2001) for phylogenetic analysis. We used the approach outlined by Huelsenbeck and Crandall (1997) to test 56 alternative models of evolution, employing PAUP* 4.0b3a and Modeltest (Posada and Crandall, 1998) discussed in detail in Harris and Crandall (2000). Once a model of evolution was chosen, it was used to estimate a tree using neighbor joining. Support for nodes was estimated using the bootstrap technique (Felsenstein, 1985), with 1000 replicates. A maximum parsimony analysis was also carried out (100 replicate heuristic search), and support for nodes estimated by bootstrapping with 1000 replicates.

\section{Results}

Including the three outgroups 57 sequences were analyzed for the mtDNA data set. Aligned sequences of the combined gene regions were $695 \mathrm{bps}$ long. The data appear to be mitochondrial DNA sequences and not nuclear integrated copies (see Nielson and Arctander, 2001) for several reasons. The protein coding gene, cytochrome $b$, contains no introns or stop codons, and the free energy of the secondary structure of the 12S rRNA is similar to the previously published sequences (data not shown; see Harris, 2001a). The extreme strand bias in the third position of the cytochrome $b$ gene is also typical in reptiles 
(A 30\% C 53\% G 04\% T 12\%, compared to average in geckos of A $36 \% \mathrm{C} 44 \% \mathrm{G} 05 \%$ T 15\%; Harris, 2001b). We concluded that the HKY model (base frequencies A 0.30 , $\mathrm{C} 0.32$, G 0.17 , T 0.21 , transition/transversion ratio 5.63), with a gamma distributed rate heterogeneity model ( 4 rate categories, $G=0.175$ ) was the most appropriate model of evolution for these data (fig. 2). Maximum parsimony analysis found 56 trees of 298 steps that were similar to the NJ trees (fig. 2). One hundred and forty five characters were parsimony informative. For the C-mos partial gene region, seven individuals were sequenced from five of the major mtDNA groups identified in this study and by Carranza et al. (2000). These were compared to the sequence of Tarentola boettgeri Steindachner, 1891 from Gran Canaria (Harris et al., 2001). A single synapomorphy united the specimens from the Cape Verde islands. One site is autapomorphic for $T$. gigas, and the other synapomorphic for T. gigas and T. rudis rudis Boulenger, 1906.

\section{Discussion}

Our results support many of the conclusions of Carranza et al. (2000). We have however included some additional taxa that were not included in the previous analysis, and which slightly alter the conclusions.

\section{Tarentola caboverdiana}

Carranza et al. (2000) included three subspecies of Tarentola caboverdiana in their analysis. Individuals of $T$. $c$. nicolauensis from São Nicolau were clearly a genetically distinct group, while $T$. c. raziana and $T$. c. substituta Joger, 1984 were reciprocally monophyletic and formed a closely related group. We included five additional specimens of T. c. nicolauensis, all of which form a monophyletic group with the previously published samples of this subspecies (98\% support). However, two of these came from the island of São Vicente (T23850 and T23851). Previously only T. c. substituta has been recorded from this island (Joger, 1993). We also included two samples of T. c. caboverdiana Schleich, 1984 from Santo Antão. These formed a distinct clade (73\% support) that is sister taxon to both $T$. c. raziana and $T$. c. substituta (73\% support). However, with the addition of two sequences of $T$. $c$. substituta and one of $T$. c. raziana, these two subspecies are no longer monophyletic with respect to each other. Therefore, within this species only three genetically distinct groups can be identified: $T$. c. nicolauensis which is not closely related to the other members of this species, $T$. $c$. caboverdiana and a closely related group of both $T$. c. raziana and $T$. c. substituta. It is not surprising that $T$. $c$. raziana and $T$. $c$. substituta are not genetically well differentiated, since they occur on islands that were probably connected during periods of lower sea levels during the last glacial periods. 


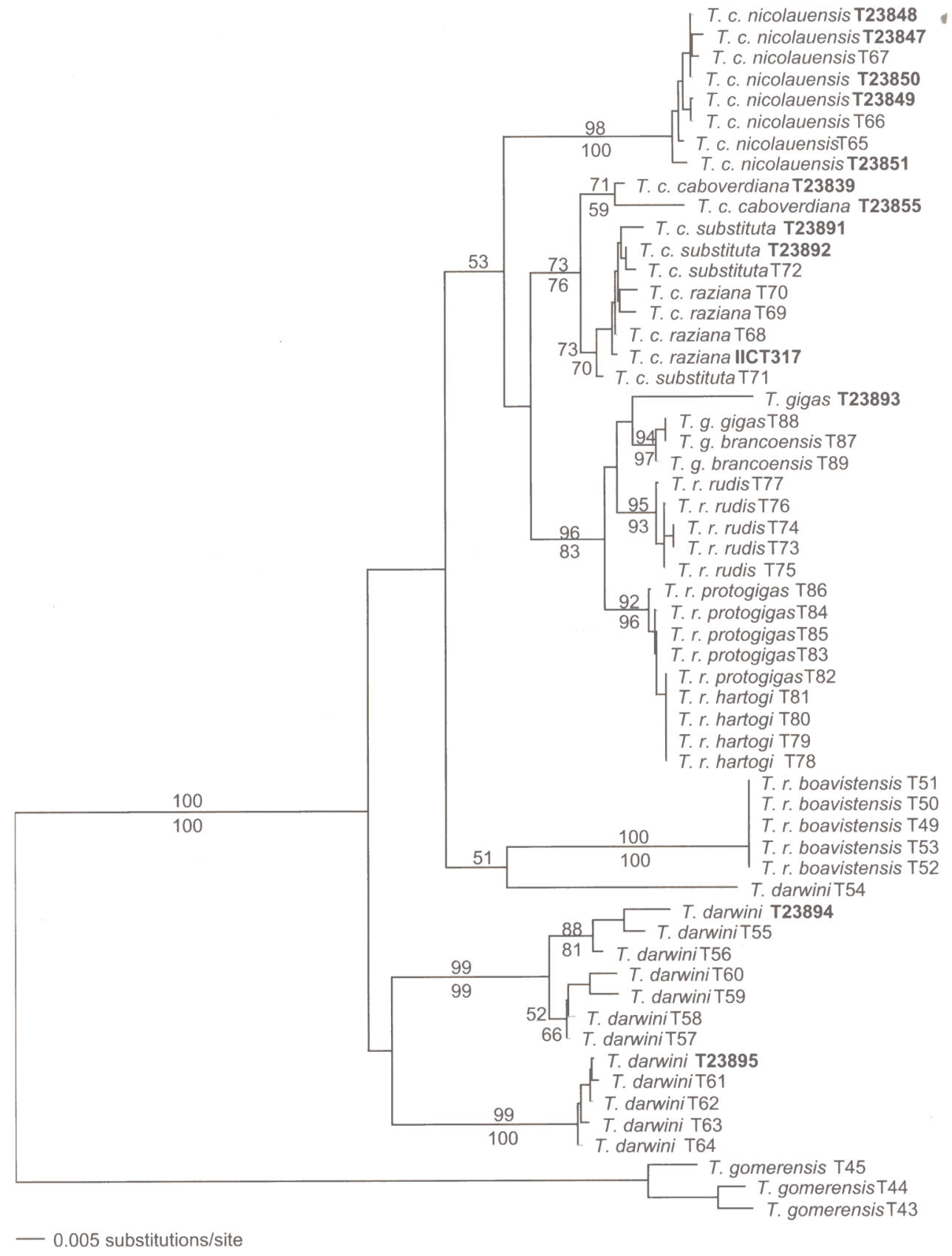

Figure 2. Tree derived from a neighbor joining analysis using the model described in the text. Bootstrap values for NJ and MP are given above and below nodes respectively. The strict consensus of the MP trees differed only in being less well resolved. Specimens new to this study have codes in bold. Others are from Carranza et al. (2000). 


\section{Tarentola gigas}

Two subspecies are currently recognised within Tarentola gigas, T. g. gigas from Raso and T. g. brancoensis Schleich, 1984 from Branco (Joger, 1993). These forms were not differentiated using mtDNA (Carranza et al., 2000). The species was reported to be confined to these small islands, although recent fossils show it was more widely distributed in the past (Carranza et al., 2000). We have included a sample from an individual of T. gigas from São Nicolau. Given that this species has not been reported from this island before, and that the sample shows considerable genetic differentiation from other T. gigas (3.3$3.6 \%$ sequence divergence with the cytochrome $b$ region) it could well represent a new subspecies. Additional sampling and detailed morphological analysis will be needed to confirm this.

\section{Phylogenetic relationships of major genetic clades}

Carranza et al. (2000) identified nine major genetic groups within Tarentola from the Cape Verde islands. Our analysis is congruent with this. Supported as a clade is a group of $T$. gigas, T. rudis rudis, T. r. hartogi Joger, 1993 and T. $r$. protogigas (96\% support). Most closely related to this group is a clade of $T$. caboverdiana caboverdiana, $T$. c. sustituta and T. c. raziana. Tarentola c. nicolauensis is the sister taxon to this whole group (53\% support). These relationships are essentially the same as those found by Carranza et al. (2000). Bootstrap supports are lower, probably because we included a shorter region of the cytochrome $b$ gene in our analysis. A single C-mos synapomorphy links $T$. gigas with $T$. rudis rudis. This is in accordance with our phylogeny estimated from mtDNA sequences.

The other taxa from the Cape Verde islands formed a separate clade in the analysis of Carranza et al. (2000), although bootstrap support was low (46\% in the NJ analysis). In our analysis T. darwini Joger, 1984 from São Nicolau and T. $r$. boavistensis Joger, 1993 are sister taxa, with $T$. darwini from Fogo and Santiago as the most basal taxa. Bootstrap support for these relationships is low, so our analysis of the available data does not resolve relationships between these groups.

\section{Community Structure}

Our analysis suggests that genetically distinct forms of Tarentola exist on more of the islands than has been previously reported. São Vicente has both $T$. caboverdiana sustituta and the genetically distinct $T$. c. nicolauensis. São Nicolau is inhabitated by $T$. gigas as well as $T$. darwini and $T$. c. nicolauensis. It seems likely that more extensive surveying will uncover additional forms on other islands. Additional data are also needed to determine if these taxa are found in sympatry on the islands. 


\section{Variation in C-mos compared to mtDNA sequences}

If both C-mos and mtDNA sequences evolve according to a molecular clock, then the relative ratio of genetic divergences between taxa will be equal in different groups. C-mos substitutions accumulate at a rate similar to that of transversion substitutions in mitochondrial protein-coding genes in passerine birds (Lovette and Bermingham, 2000). In Mabuya skinks from the Cape Verde islands, taxa show considerably lower levels of mtDNA substitutions than geckos, but eleven C-mos substitutions were detected (Brehm et al., 2001), as opposed to two within Tarentola from the islands in this study. Geckos on average have higher levels of mtDNA divergence between species than other lizards (Harris, 2001a). This could be an artifact of taxonomy, for example geckos could simply be morphologically more conservative so that some species have been overlooked. However, if C-mos variation is low between gecko species, this implies they may have a higher rate of mtDNA divergence than other lizards, which would affect molecular clock calibrations currently used. Additional sampling of taxa for both mtDNA and C-mos will be needed to study this possibility further.

\section{Conclusions}

Despite the extensive sampling of Carranza et al. (2000) we have uncovered genetically distinct forms on islands where they have not previously been recorded. This knowledge is essential for future conservation of these unique geckos. Our study suggests that even more sampling will be necessary to fully understand the evolutionary history of the group and their systematics.

Acknowledgements. We are grateful to L. Carvalho of the Secretaria Executiva para o Ambiente from the Cape Verde government and ICCTI of Lisbon for assistance in funding surveys in the Cape Verde Islands, and to Dr Margarida Pinheiro (Center of Zoology, IICT, Lisbon) for providing the sample from Raso. Thanks also to G. Gollmann and two anonymous reviewers who gave constructive criticisms on an earlier draft of this manuscript.

\section{References}

Brehm, A., Jesus, J., Pinheiro, M., Harris, D.J. (2001): Relationships of Scincid lizards Mabuya (Reptilia: Scincidae) from the Cape Verde Islands based on mitochondrial and nuclear DNA sequences. Mol. Phylogenet. Evol. 19: 311-316.

Carranza, S., Arnold, E.N., Mateo, J.A., Lopez-Jurado, L.F. (2000): Long-distance colonization and radiation in gekkonid lizards, Tarentola (Reptilia: Gekkonidae), revealed by mitochondrial DNA sequences. Proc. Roy. Soc. Lond. B 267: 637-649,

Carranza, S., Arnold, E.N., Mateo, J.A., Lopez-Jurado, L.F. (2001): Parallel gigantism and complex colonization patterns in the Cape Verde scincid lizards Mabuya and Macroscincus (Reptilia: Scincidae) revealed by mitochondrial DNA sequences. Proc. Roy. Soc. Lond. B 268: 1595-1603.

Felsenstein, J. (1985): Confidence limits on phylogenies: an approach using the bootstrap. Evolution 39: 783-791. 
Harris, D.J. (2001a): Re-evaluation of $16 \mathrm{~S}$ Ribosomal RNA variation in Bufo (Anura: Amphibia). Mol. Phylogenet. Evol. 19: 326-329.

Harris, D.J. (2001b): Reassessment of comparative genetic distance in reptiles from the mitochondrial cytochrome $b$ gene. Herpetol. J. in press.

Harris, D.J., Arnold, E.N., Thomas, R.H. (1998): Relationships of the lacertid lizards (Reptilia: Lacertidae) estimated from mitochondrial DNA sequences and morphology. Proc. R. Soc. London B 265: 1939-1948.

Harris, D.J., Crandall, K.A. (2000): Intragenomic variation within ITS1 and ITS2 of crayfish (Decapoda, Cambaridae): implications for phylogenetic and microsatellite studies. Mol. Biol. Evol. 17: 284-291.

Harris, D.J., Marshall, J.C., Crandall, K.A. (2001): Squamate relationships based on C-mos nuclear DNA sequences: increased taxon sampling improves bootstrap support. Amphibia-Reptilia 22: 235-242.

Huelsenbeck, J.P., Crandall, K.A. (1997): Phylogeny estimation and hypothesis testing using maximum likelihood. Ann. Rev. Ecol. Syst. 28: 437-466.

Jesus, J., Brehm, A., Pinheiro, M., Harris, D.J. (2001): Relationships of Hemidactylus (Reptilia: Gekkonidae) from the Cape Verde Islands: what mitochondrial DNA data indicate. J. Herpetology, in press.

Joger, U. (1984): Die Radiation der Gattung Tarentola in Makaronesien. Courier Forsch.-Inst. Senckenberg 71: 91-111.

Joger, U. (1993): On two collections of reptiles and amphibians from the Cape Verde Islands, with descriptions of three new taxa. Courier Forsch.-Inst. Senckenberg 159: 437-444.

Kocher, T.D., Thomas, W.K., Meyer, A., Edwards, S.V., Pääbo, S., Villablanca, F.X., Wilson, A.C. (1989): Dynamics of mitochondrial DNA evolution in animals: amplification and sequencing with conserved primers. Proc. Nat. Acad. Sci. USA 86: 6196-6200.

Lovette, I.J., Bermingham, E. (2000): C-mos variation in Songbirds: molecular evolution, phylogenetic implications and comparisons with mitochondrial differentiation. Mol. Biol. Evol. 17: 1569-1577.

Mitchell-Thome, R.C. (1983): Radiometric studies in Macronesia. Bol. Mus. Mun. Funchal. 37: 52-85.

Nielsen, K.K., Arctander, P. (2001): Recombination among multiple mitochondrial pseudogenes from a passerine genus. Mol. Phylogenet. Evol. 18: 362-369.

Posada, D., Crandall, K.A. (1998): Modeltest: testing the model of DNA substitution. Bioinformatics 14: 817818.

Saint, K.M., Austin, C.C., Donnellan, S.C., Hutchinson, M.N. (1998): C-mos, a nuclear marker useful for squamate phylogenetic analysis. Mol. Phylogenet. Evol, 10: 259-263.

Schleich, V. (1987): Herpetofauna Caboverdiana. Spixiana 12: 1-75.

Swofford, D.L. (2001): PAUP*: Phylogenetic Analysis Using Parsimony (and other methods) 4.0.b5. Sunderland, Massachusetts, USA, Sinauer Associates.

Thompson, J.D., Higgins, D.G., Gibson, T.J. (1994): Clustal W: improving the sensitivity of progressive multiple sequence alignment through sequence weighting, position specific gap penalties and weight matrix choice. Nucl. Acid. Res. 22: 4673-4680.

Received: May 11, 2001. Accepted: July 30, 2001. 